\title{
Influence of gold-silica nanoparticles on the performance of small-molecule bulk heterojunction solar cells
}

\author{
Xiaoyan $\mathrm{Xu}^{\mathrm{a}}$, Aung Ko Ko Kyaw ${ }^{\mathrm{b}}$, Bo Peng ${ }^{\mathrm{c}}$, Qihua Xiong ${ }^{\mathrm{c}}$, Hilmi Volkan Demir ${ }^{\mathrm{a}, \mathrm{c}, \mathrm{d}}$, Ye Wang ${ }^{\mathrm{e}}$, \\ Terence K.S. Wong ${ }^{\mathrm{a}, *}$, Xiao Wei Sun ${ }^{\mathrm{a}, *}$ \\ a School of Electrical and Electronic Engineering, Nanyang Technological University, Singapore 639798, Singapore \\ ${ }^{\mathrm{b}}$ Institute of Materials Research and Engineering (IMRE), Agency for Science Technology and Research (A*STAR), Singapore 117602, Singapore \\ 'School of Physical and Mathematical Sciences, Nanyang Technological University, Singapore 639798, Singapore

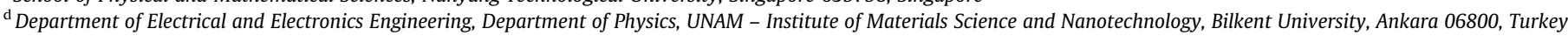 \\ e Pillar of Engineering Product Development, Singapore University of Technology and Design, 8 Somapeh Road, Singapore 487372, Singapore
}

\section{A R T I C L E I N F O}

\section{Article history:}

Received 10 December 2014

Received in revised form 4 March 2015

Accepted 16 March 2015

Available online 18 March 2015

\section{Keywords:}

Small-molecule solar cell

Nanosphere

Nanorod

Light trapping

Plasmonic effect

Stability

\begin{abstract}
A B S T R A C T
Light trapping by gold $\mathrm{Au}$ )-silica nanospheres and nanorods embedded in the active layer of small-molecule (SM) organic solar cell has been systematically compared. Nanorod significantly outperforms nanosphere because of more light scattering and higher quality factor for localized surface plasmon resonance (LSPR) triggered by nanorods. The optimum concentration of nanorod was characterized by charge carrier transport and morphology of the active layers. At optimum nanorod concentration, almost no change in the morphology of the active layer reveals that LSPR and scattering effects rather than the morphology are mainly responsible for the enhanced power conversion efficiency. In addition, the preliminary lifetime studies of the SM solar cells with and without Au-silica nanorods were conducted by measuring the current density-voltage characteristics over 20 days. The results show that plasmonic device with nanorods has no adverse impact on the device stability.
\end{abstract}

(c) 2015 Elsevier B.V. All rights reserved.

\section{Introduction}

Solution-processed small-molecule (SM) donors have stimulated much interest as a viable alternative to the widely studied conjugated polymers for organic bulk heterojunction (BHJ) solar cells [1-4]. Solution-processed SM donors have the advantage over conjugated polymer counterparts since the SM donor molecules are monodisperse and can be purified using reproducible fabrication protocols and therefore provide more uniform performance in SM-based devices [5,6].

Similar to polymer solar cells (PSCs), SM solar cells have major limitation in insufficient sunlight absorption because the active layer of SM solar cells should be very thin $(\sim 100 \mathrm{~nm})$ due to low carrier mobility and short exciton diffusion length [7-9]. Hence, there is a need to capture more light without increasing the thickness of the BHJ layer. As discussed in a recent review [10], the light trapping techniques for thin film organic solar cells include the cells with V-shaped (folded) geometry, incoupler at the front surface, structured back reflector and substrates. In addition, in recent

\footnotetext{
* Corresponding authors.

E-mail addresses: ekswong@ntu.edu.sg (T.K.S. Wong), EXWSun@ntu.edu.sg (X.W. Sun).
}

years, there has been intense interest in applying the plasmonic properties of metallic nanoparticles (NPs) to enhance the performance of PSCs by doping these NPs directly into the active layer [11-14]. Typically, most existing plasmonic materials for improved light absorption in PSCs are focused on the spherical NPs with a diameter less than $50 \mathrm{~nm}$ [15-18]. Here, a near field effect attributed to the enhancement of local electric field around the NP is thought to enhance the optical absorption in the BHJ. However, the localized surface plasmon resonance (LSPR) spectrum of these NPs has only one LSPR peak in the shorter wavelength region. In addition, the small size and spherical shape in these NPs greatly weakens the light scattering of NPs inside of PSCs [19]. Recently, the incorporation of large-sized nanorods into the low bandgap PSCs was reported to result in $26 \%$ relative improvement in power conversion efficiency (PCE) [20]. This enhancement was due to far field scattering by the incorporated nanorods increasing the optical path length within the cell. Despite this enhancement in PSCs, the influence of the nanorods on PSCs has not been explored in sufficient detail. In a recent paper by Wadam et al. [21], it was shown that the PCE enhancement mechanism in poly(3-hexylthiophene) (P3HT): fullerene BHJ cells incorporated with Au nanorods was not due to plasmonic effects but rather by a favorable modification of the BHJ nano-morphology by the nanorods. 
In this paper, we incorporated core-shell structured Au-silica NPs into SM solar cells based on 7,7'-(4,4-bis(2-ethylhexyl)-4Hsilolo[3,2-b:4,5-b']-dithiophene-2,6-diyl)bis(6-fluoro-4-(5'-hexyl-[2, 2'-bithiophen]-5-yl)benzo[c][1,2,5]thiadiazole):[6,6]-phenyl- $\mathrm{C}_{71^{-}}$butyric acid methyl ester (p-DTS(FBTTh $\left.\left.{ }_{2}\right)_{2}: \mathrm{PC}_{70} \mathrm{BM}\right)$. The two different geometries of NPs, namely nanorod and nanosphere were examined and the geometry effect on SM devices were studied by comparing total light scattering and the quality factor for LSPR. In addition, the X-ray diffraction (XRD) and surface morphology measurements at the optimized Au-silica nanorod concentration were conducted to verify that the SM BHJ structure does not change with the Au-silica nanorods unlike P3HT system [21]. The experimental results confirm that for the p-DTS(FBTTh $)_{2}: \mathrm{PC}_{70} \mathrm{BM}$ system, light scattering and LSPR effect rather than morphology of the BHJ layer contribute to the enhancement of PCE in plasmonic SM device. Moreover, the charge carrier transport characterization and atomic force microscopy (AFM) were used to identify the factors that determine the optimum concentration of nanorods in plasmonic devices. Finally, the impact of nanorods on the stability of SM device was also examined.

\section{Experimental}

\subsection{Synthesis of nanorods and nanospheres}

We synthesized Au-silica core-shell nanorods/nanospheres, using the seed-mediated method [20,22]. The procedure of Au-silica nanorods consists of two parts: (i) seed-mediated synthesis of gold nanorods using a seed solution and the reagent hexadecyltrimethylammonium bromide and (ii) solution synthesis of the silica shell using tetraethyl orthosilicate [23]. In order to have a more rigorous comparison with core-shell gold nanorods, bare gold nanorods were also synthesized by the seed-mediated method only.

\subsection{Device fabrication}

SM BHJ solar cells were fabricated by the following process. Indium tin oxide (ITO)-coated glass substrates were cleaned by detergent, deionized water, acetone and isopropyl alcohol sequentially in an ultrasonic bath. Each substrate was blow dried by nitrogen and treated with oxygen plasma for $10 \mathrm{~min}$ to reform the ITO surface. Poly(3,4-ethylene-dioxythiophene):poly (styrenesulphonate)(PEDOT:PSS) (Baytron P 4083) was spin-coated at $5000 \mathrm{rpm}$ for $40 \mathrm{~s}$ on the ITO-coated substrate to obtain a thickness of $30 \mathrm{~nm}$. The PEDOT:PSS film was then annealed at $150{ }^{\circ} \mathrm{C}$ for $20 \mathrm{~min}$ on a hotplate. The SM BHJ solutions were prepared by adding $35 \mathrm{mg}$ of p-DTS(FBTTh $)_{2}: \mathrm{PC}_{70} \mathrm{BM}$ blend with a weight ratio of 3:2 and Au-silica (or Au) NPs into $1 \mathrm{ml}$ of chlorobenzene (CB) with $0.4 \mathrm{v} / \mathrm{v} \% 1,8$ octanedithiol processing additive. The SM BHJ solutions were spin-casted at $2000 \mathrm{rpm}$ for $45 \mathrm{~s}$ on the surface of PEDOT:PSS layer and then thermally annealed at $80^{\circ} \mathrm{C}$ for $10 \mathrm{~min}$ to remove residual solvent in a glove box. All SM BHJ films have the thicknesses of $\sim 100 \mathrm{~nm}$ measured by surface profiler. Finally, the cathode consisting of $20 \mathrm{~nm} \mathrm{Ca}$ and $80 \mathrm{~nm} \mathrm{Ag}$ was thermally deposited under vacuum condition of $1 \times 10^{-4} \mathrm{~Pa}$. All fabricated devices with an active area of $8 \mathrm{~mm}^{2}$ were encapsulated using epoxy and cover glass before removal from the glove box.

For the charge transport studies, hole-only devices were fabricated with a structure of the ITO/Molybdenum trioxide $\left(\mathrm{MoO}_{3}\right) / \mathrm{p}-\mathrm{DTS}\left(\mathrm{FBTTh}_{2}\right)_{2}: \mathrm{PC}_{70} \mathrm{BM} / \mathrm{MoO}_{3} / \mathrm{Ag}$. The second layer of $\mathrm{MoO}_{3}$ blocks the injection of electrons from the $\mathrm{Ag}$ electrode. Electron-only devices have a structure of ITO/Al/p-DTS $\left(\mathrm{FBTTh}_{2}\right)_{2}: \mathrm{PC}_{70} \mathrm{BM} / \mathrm{Ca} / \mathrm{Al}$. The first layer of $\mathrm{Al}$ blocks the injection of holes from the ITO electrode.

\subsection{Device characterization}

The dimensions of the Au-silica NPs were characterized by a JEOL 1400 transmission electron microscope (TEM) at an accelerating voltage of $100 \mathrm{kV}$. The height and phase images of the pDTS $\left(\mathrm{FBTTh}_{2}\right)_{2}: \mathrm{PC}_{70} \mathrm{BM}$ films with and without nanorods were scanned in the tapping mode using a Cypher S AFM (Asylum Research). XRD measurements were conducted using D8 Advance Eco X-ray diffractometer at $40 \mathrm{keV}$ and $25 \mathrm{~mA}$ using $\mathrm{Cu} \mathrm{K} \alpha$ $(\lambda=1.54 \AA)$.

Optical absorption spectra of the BHJ layers were measured using the Perkin Elmer Lambda 950 spectrophotometer which is fitted with a $150 \mathrm{~mm}$ diameter integrating sphere. For absorption measurement, the reflectance and specular exclusion ports of the integrating sphere are blocked by white standard plates. By unblocking these two ports consecutively, the ratio of diffuse scattering to total scattering or the haze factor could also be measured. Prior to absorption measurements, the film thickness of all layers was measured by a KLA-Tencor P-10 surface profiler to ensure the thicknesses are the same.

For solar cell characterization, current density-voltage $(J-V)$ characteristics under dark and illuminated conditions were measured with a Keithley 2400 source meter unit. A solar simulator (Solar Light Inc.) provides simulated sunlight with AM1.5G spectrum and irradiance of $100 \mathrm{~mW} / \mathrm{cm}^{2}$. The external quantum efficiency (EQE) spectra were conducted by a Bentham PVE 300 system consisting of a calibrated silicon photodiode, a xenon lamp, monochromator, lock-in amplifier and chopper wheel.

\section{Results and discussion}

\subsection{Comparison of Au-silica nanospheres and nanorods}

TEM images in the insets of Fig. 1 display that the average diameter of nanospheres core and the thickness of the silica shell are $\sim 25 \mathrm{~nm}$ and $\sim 5 \mathrm{~nm}$ respectively. The average diameter and length of the Au nanorods core are $\sim 31 \mathrm{~nm}$ and $\sim 86 \mathrm{~nm}$ respectively and the thickness of the silica shell is $\sim 5 \mathrm{~nm}$. The histograms of TEM image measurements used to determine these average NP size parameters can be found in the Supplementary information. A $5 \mathrm{~nm}$ shell thickness is chosen because as reported recently [24], significant enhanced localized field should exist outside the shell

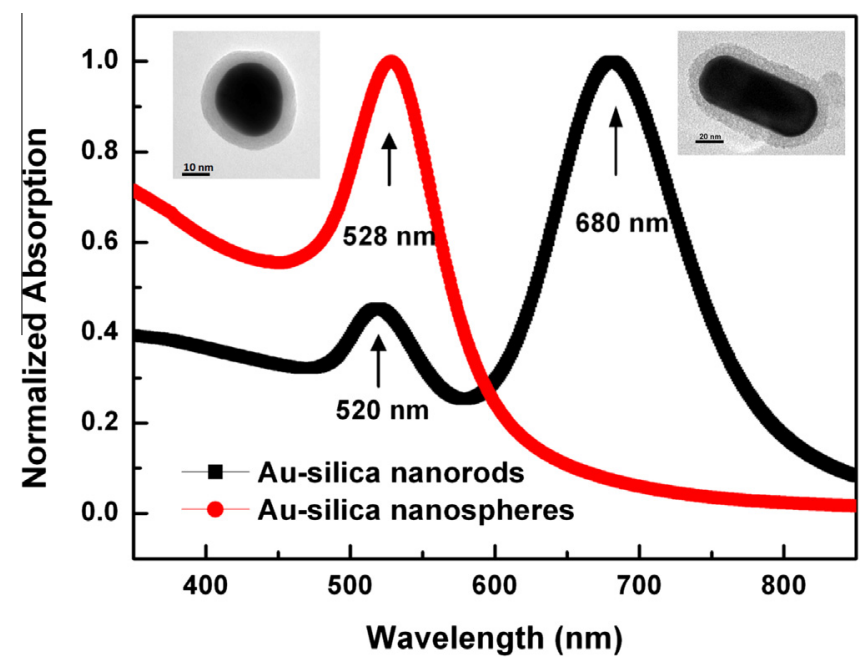

Fig. 1. Normalized absorption spectra of the Au-silica nanorods and nanospheres in CB. The inset is the TEM images of Au-silica nanosphere and nanorod. The scale bars represent $10 \mathrm{~nm}$ and $20 \mathrm{~nm}$ respectively. 

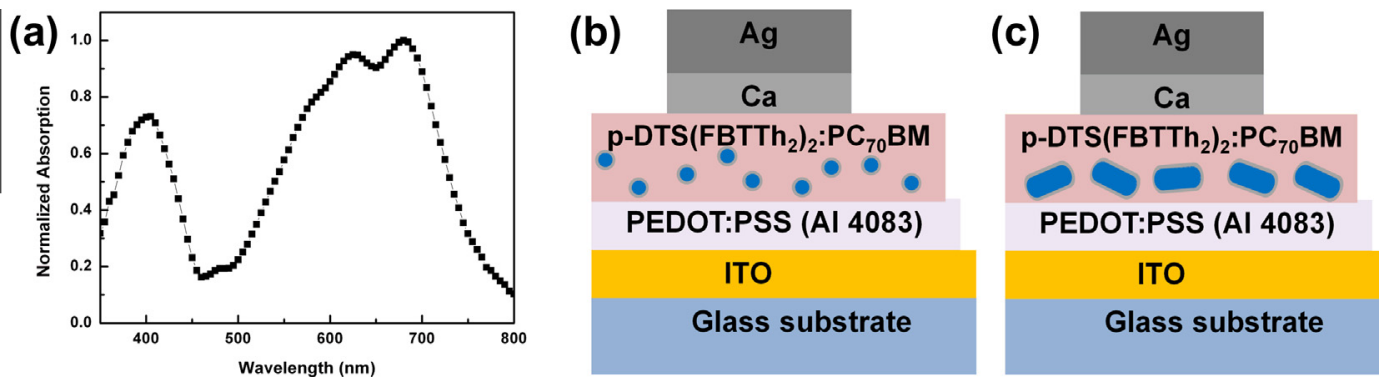

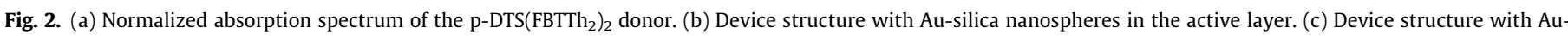
silica nanorods in the active layer.
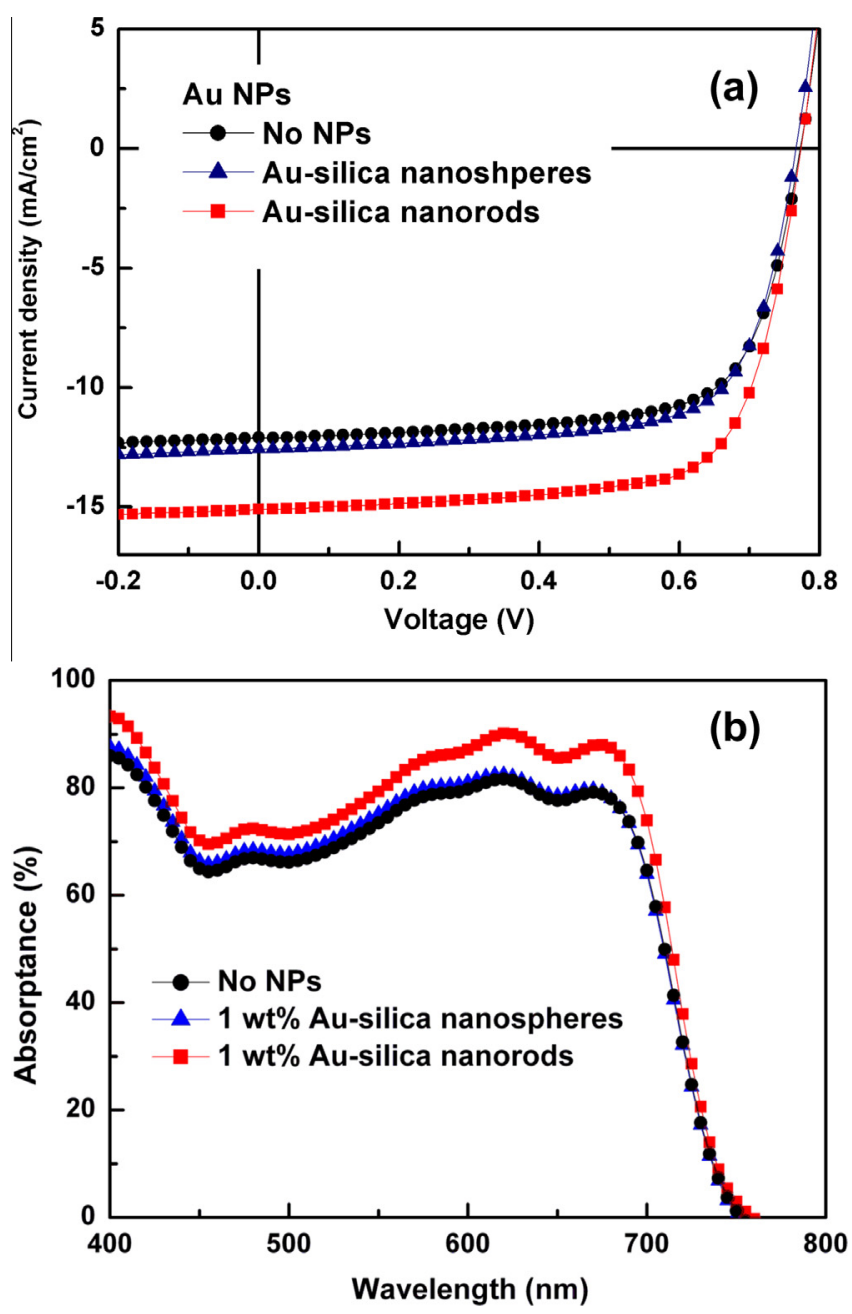

Fig. 3. (a) $J-V$ characteristics and (b) optical absorption spectra of pDTS $\left(\text { FBTTh }_{2}\right)_{2}: \mathrm{PC}_{70} \mathrm{BM}$ BHJ solar cells with and without Au NPs.

and surface recombination can be avoided. The normalized absorption spectra indicate that Au-silica nanospheres in $\mathrm{CB}$ have only one LSPR peak at $528 \mathrm{~nm}$, whereas Au-silica nanorods in CB have dual LSPR peaks at $520 \mathrm{~nm}$ and $680 \mathrm{~nm}$. Unlike the case of P3HT, the major LSPR peak of nanorods at $680 \mathrm{~nm}$ well matches the absorption peak of p-DTS(FBTTh $)_{2}$ donor as shown in Fig. 2a. SM $\mathrm{BHJ}$ solar cells were fabricated with the device structure of ITO/ PEDOT:PSS/p-DTS(FBTTh $)_{2}: \mathrm{PC}_{70} \mathrm{BM} / \mathrm{Ca} / \mathrm{Ag}$. Either Au-silica nanospheres or Au-silica nanorods was blended directly into the pDTS(FBTTh $)_{2}:$ PC $_{70}$ BM films of SM solar cells (Fig. 2b and c).
The $J-V$ characteristics under AM1.5G irradiation at $100 \mathrm{~mW} /$ $\mathrm{cm}^{2}$ were conducted for SM devices with and without Au-silica nanospheres/nanorods (Fig. 3a). The concentrations of Au-silica nanospheres and nanorods were optimized at $1 \%$ weight ratio (wt\%). The reference device without NPs has an average PCE of $6.5 \pm 0.14 \%$ with the short-circuit current density $\left(J_{S C}\right)$ of $12.04 \pm 0.27 \mathrm{~mA} / \mathrm{cm}^{2}$, open-circuit voltage $\left(V_{O C}\right)$ of $0.77 \pm 0.01 \mathrm{~V}$, and fill factor $(F F)$ of $70.2 \pm 0.65 \%$. Upon incorporation of only Ausilica nanospheres, there is a slight increase to $12.69 \pm 0.24 \mathrm{~mA} /$ $\mathrm{cm}^{2}$ in $J_{S C}$ and no changes in the $V_{O C}$ and FF. When only Au-silica nanorods were embedded in SM device, the $J_{S C}$ was largely
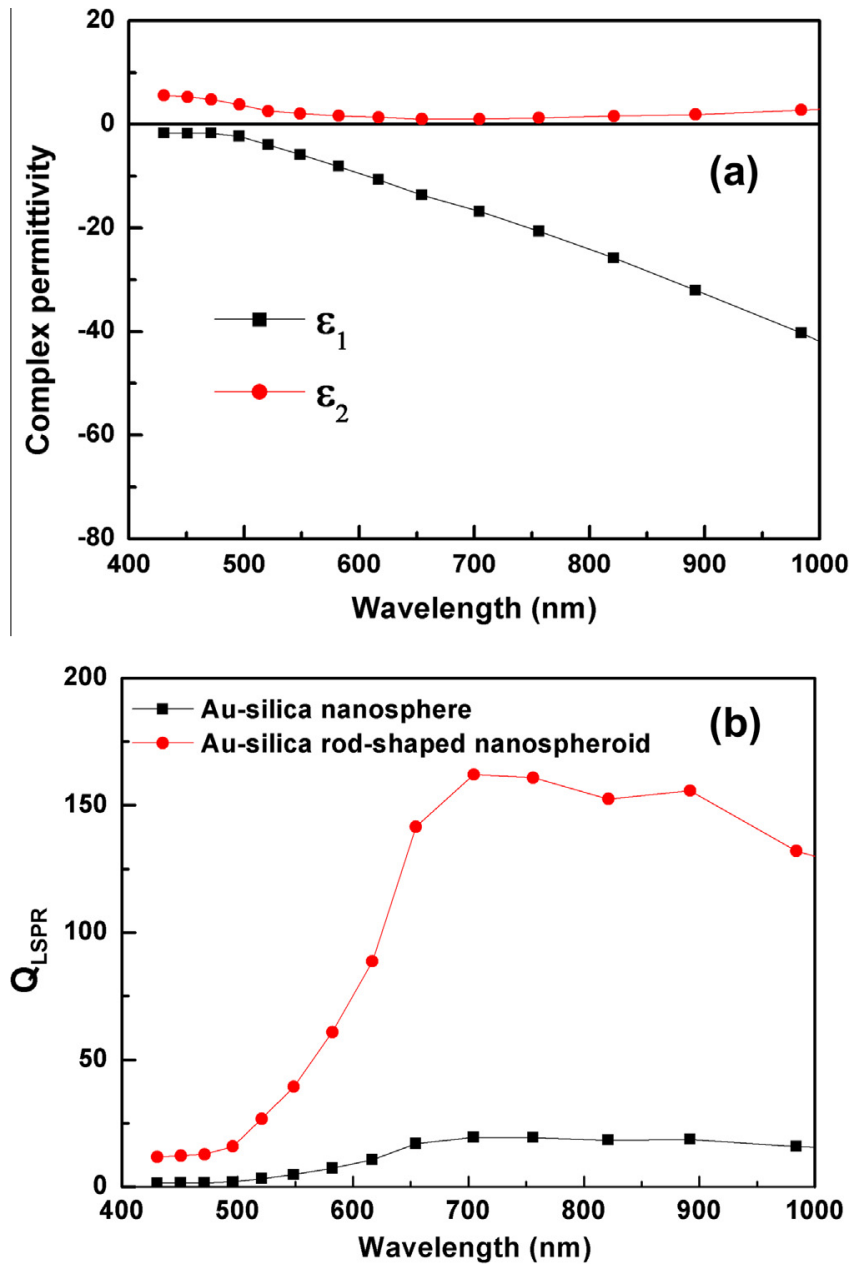

Fig. 4. (a) The real and imaginary parts of the complex dielectric function for Au. (b) Quality factor for LSPR $\left(Q_{L S P R}\right)$ for Au-silica sphere and Au-silica rod-shaped spheroid. 

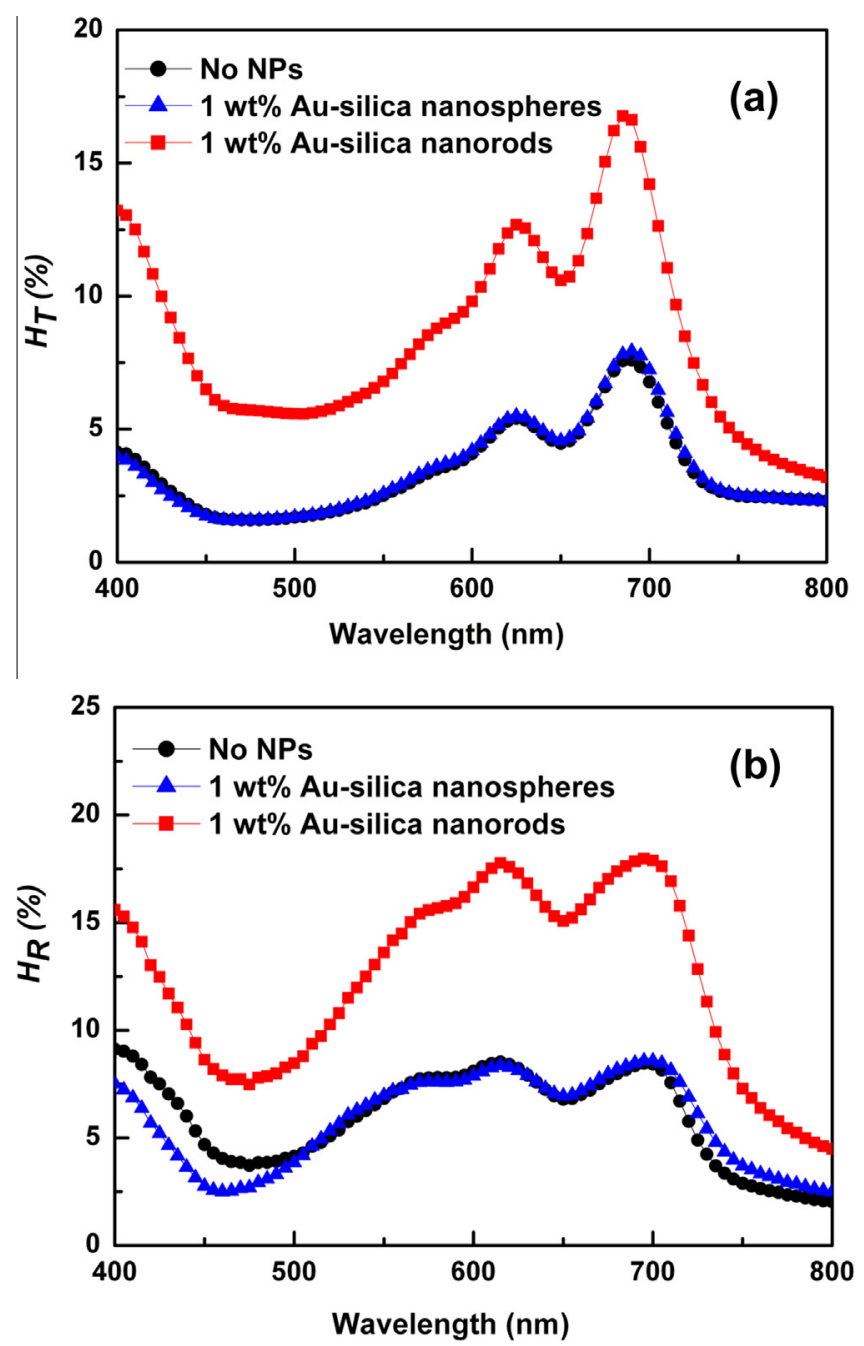

Fig. 5. (a) Transmittance haze factor $\left(H_{T}\right)$ and (b) reflection haze factor $\left(H_{R}\right)$ spectra of the p-DTS(FBTTh $)_{2}: \mathrm{PC}_{70} \mathrm{BM}$ films with and without Au NPs.

increased to $15.14 \pm 0.35 \mathrm{~mA} / \mathrm{cm}^{2}$ while the $V_{O C}$ and $F F$ remained the same. Such larger enhancement in $J_{S C}$ is because the absorption enhancement of the BHJ film with Au-silica nanorods is much larger than that of $\mathrm{BHJ}$ film with Au-silica nanospheres relative to the bare $\mathrm{BHJ}$ film as shown in Fig. 3b. The absence of change in $V_{O C}$ upon incorporation of Au-silica nanorods into the BHJ of the SM device is different from what was reported in [25] where bare Au NPs were introduced into poly(3-hexylthophene):PCBM. We attribute the invariant $V_{O C}$ in our SM devices to the fact that an interface dipole layer is not formed between $\mathrm{Au}$ and the $\mathrm{BHJ}$ when a silica shell is present and as a result, there is no shift in the energy levels of the donor [25].

The $J-V$ characteristics of SM devices with and without bare Au nanorods can be found in the Supplementary information. Fig. S1 represents the $J-V$ characteristics of the p-DTS(FBTTh $)_{2}: \mathrm{PC}_{70} \mathrm{BM}$ devices with various bare $\mathrm{Au}$ nanorod concentrations under AM1.5G irradiation at $100 \mathrm{~mW} / \mathrm{cm}^{2}$. The photovoltaic parameters for each concentration are listed in Table S1. The reference device without Au NPs has an average PCE of $6.5 \pm 0.14 \%$ with an $V_{O C}$ of $0.77 \pm 0.01 \mathrm{~V}, \quad$ a $J_{S C}$ of $12.04 \pm 0.27 \mathrm{~mA} / \mathrm{cm}^{2}$ and a $F F$ of $70.2 \pm 0.65 \%$. After incorporation of the bare Au nanorod with the concentration of $0.25 \mathrm{wt} \%$, the $J_{S C}$ and $V_{O C}$ increased to $12.58 \pm 0.35 \mathrm{~mA} / \mathrm{cm}^{2}$ and $0.78 \pm 0.01 \mathrm{~V}$ while the $F F$ has almost no change. The enhanced $J_{S C}$ is ascribed to the light absorption induced by plasmonic effect of Au nanorods. The very small
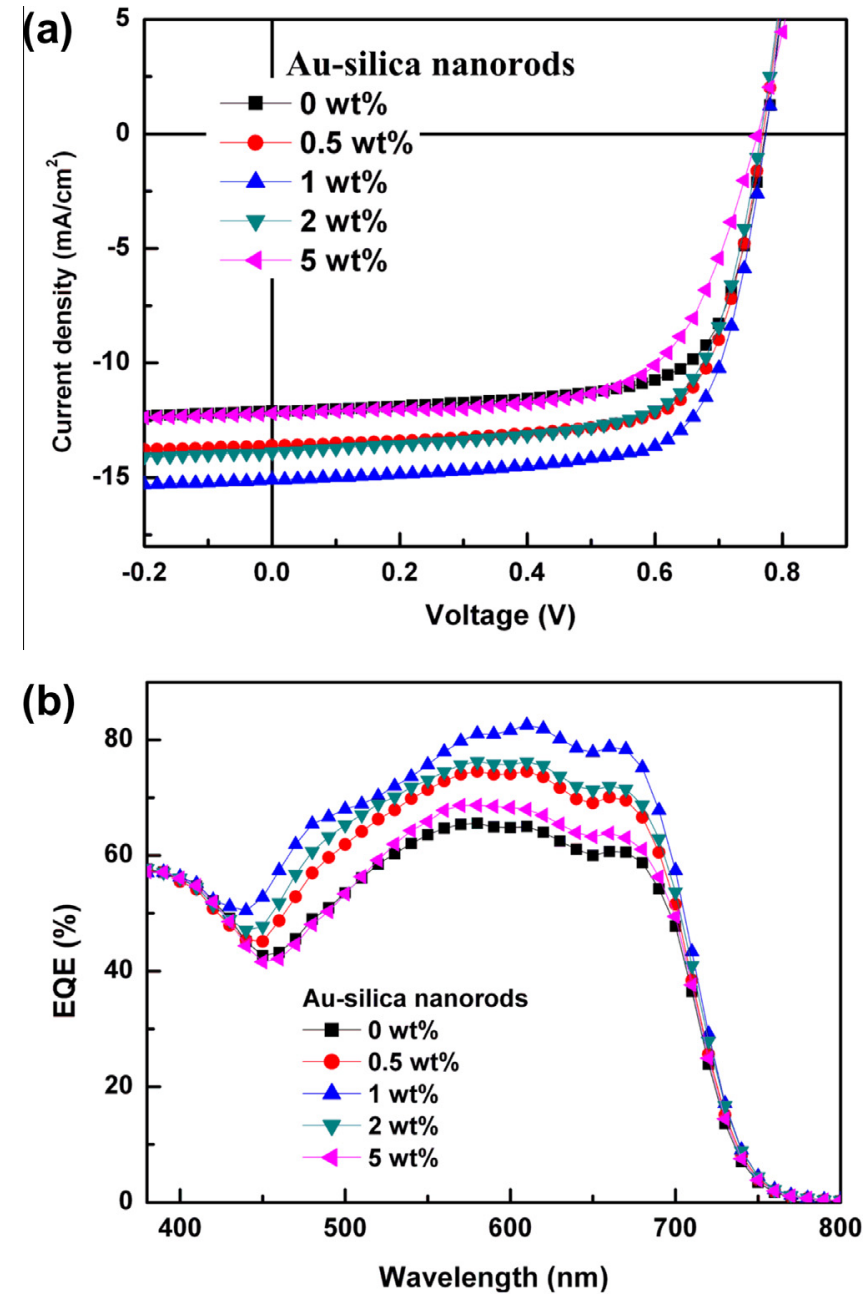

Fig. 6. (a) $J-V$ characteristics and (b) EQE spectra of p-DTS(FBTTh $)_{2}: \mathrm{PC}_{70} \mathrm{BM} \mathrm{BHJ}$ solar cells with Au-silica nanorod concentrations of $0 \mathrm{wt} \%, 0.5 \mathrm{wt} \%, 1 \mathrm{wt} \%, 2 \mathrm{wt} \%$ and $5 \mathrm{wt} \%$.

Table 1

Photovoltaic parameters of SM BHJ devices based on p-DTS(FBTTh $)_{2}: \mathrm{PC}_{70} \mathrm{BM}$ with different Au-silica nanorod concentrations under $100 \mathrm{~mW} / \mathrm{cm}^{2}$ AM1.5G simulated solar irradiation.

\begin{tabular}{lllll}
\hline $\begin{array}{l}\text { Au NPs } \\
\text { concentration (wt\%) }\end{array}$ & $V_{O C}(\mathrm{~V})$ & $J_{S C}\left(\mathrm{~mA} / \mathrm{cm}^{2}\right)$ & $F F(\%)$ & PCE (\%) \\
\hline 0 & $0.77 \pm 0.01$ & $12.04 \pm 0.27$ & $70.2 \pm 0.65$ & $6.5 \pm 0.14$ \\
0.5 & $0.77 \pm 0.01$ & $13.62 \pm 0.30$ & $70.5 \pm 0.84$ & $7.4 \pm 0.15$ \\
1 & $0.77 \pm 0.01$ & $15.14 \pm 0.35$ & $70.4 \pm 0.61$ & $8.2 \pm 0.12$ \\
2 & $0.77 \pm 0.01$ & $13.93 \pm 0.40$ & $68.4 \pm 0.80$ & $7.3 \pm 0.24$ \\
5 & $0.76 \pm 0.01$ & $12.08 \pm 0.34$ & $65.8 \pm 0.88$ & $6.0 \pm 0.17$ \\
\hline
\end{tabular}

improvement of $0.01 \mathrm{~V}$ in the $V_{O C}$ may be related to the small energy barrier for hole extraction as shown in Fig. S2 and the work function of $\mathrm{Au}$ is almost the same as the HOMO of the donor [25]. The PCE in the device with the $0.25 \mathrm{wt} \%$ of bare nanorod reaches $6.9 \pm 0.15 \%$. When the bare nanorod concentration further increased from $0.25 \mathrm{wt} \%$ to $1 \mathrm{wt} \%$, the PCE, $J_{S C}, F F$ and $V_{O C}$ all declined. A decrease in $V_{O C}$ has also been observed when bare $\mathrm{Ag}$ nanoparticles were added at the interface between the hole transport layer and the BHJ of a PSC [26]. We attribute the decrease in photovoltaic parameters in our devices to increased charge carrier recombination at the surface of the bare Au nanorods which creates the internal shunts within the BHJ active layer [27]. The charge carrier recombination induced by bare Au nanorods was confirmed 
by light intensity dependence of short-circuit current density (see Section 3.2). Another possible reason is the increased quenching of excitons upon incorporation of $\mathrm{Au}$ [26]. Therefore, there is a compromise between the charge carrier recombination and plasmonic effect in the device embedded with the bare Au nanorod. Finally, the best performance with the PCE of $6.9 \pm 0.15 \%$ in device with the bare nanorod is much lower than that with the maximum PCE of $8.2 \pm 0.12 \%$ in device with the Au-silica nanorod, which indicated that the device performance can indeed be significantly improved by incorporation of Au-silica nanorods with shell thickness of $5 \mathrm{~nm}$, compared to the bare Au nanorods.

The two main mechanisms of light absorption enhancement in these plasmonic organic solar cells (OSCs) are the LSPR effect and scattering effect since metallic NPs upon the excitation of incident light can enhance not only the localized electric field intensity but also scatter light into longer optical path length within the active layer [28]. For LSPR effect, the electromagnetic simulations for Au nanosphere and Au nanosphere embedded in the OSCs have been reported separately in the previous publications [27,29]. The enhanced electric field can exist outside the Au nanosphere and $\mathrm{Au}$ nanorod. However, the quantitative comparison of the local field enhancement for these different plasmonic nanostructures in the OSCs has not been reported yet. It can be made using the quality factor $Q_{f}$ defined in general as the ratio of the enhanced
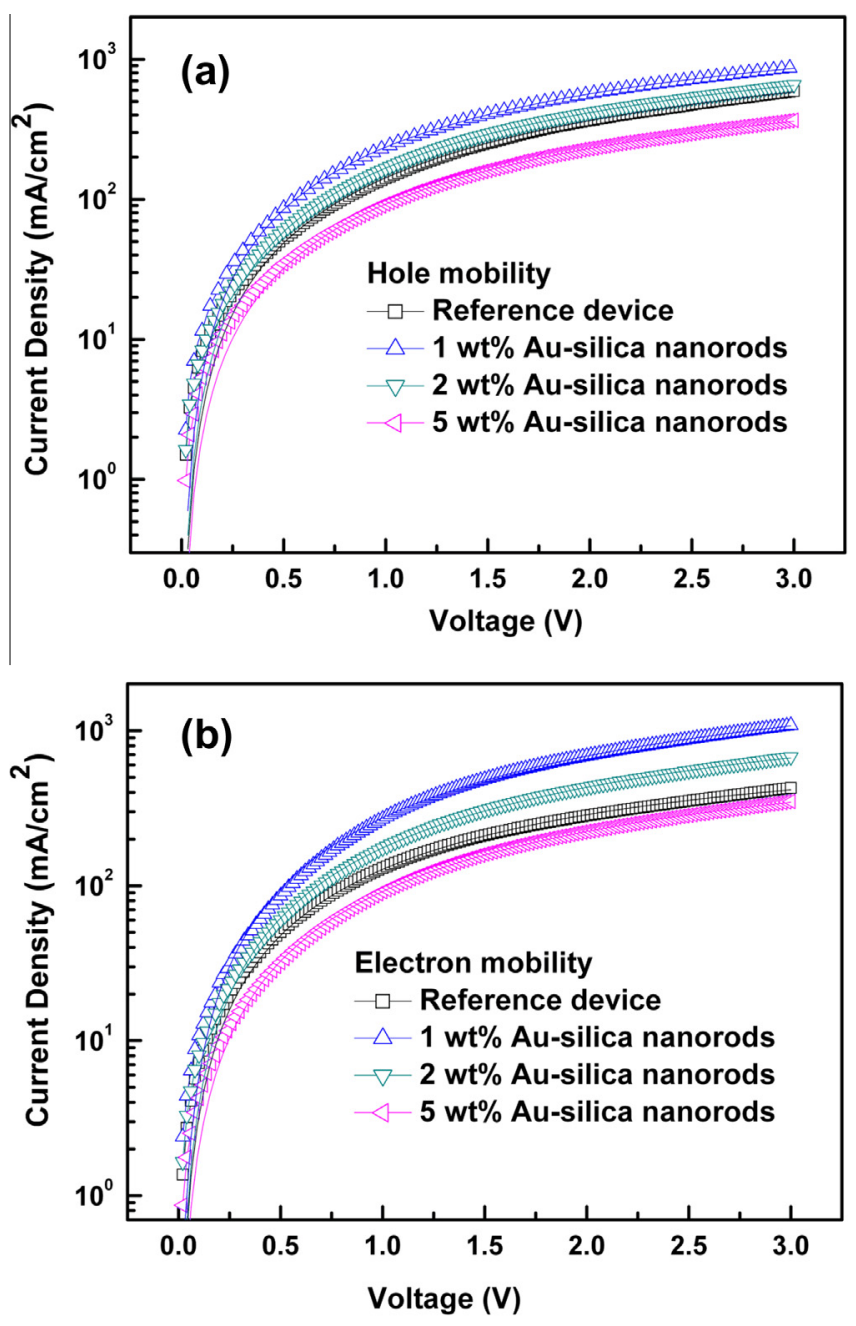

Fig. 7. The dark $J-V$ characteristics of (a) hole-only devices (b) and electron-only devices with Au-silica nanorod concentrations of $0 \mathrm{wt} \%, 1 \mathrm{wt} \%, 2 \mathrm{wt} \%$ and $5 \mathrm{wt} \%$. The solid lines represent the best fitting using the SCLC model.
Table 2

Zero-field hole and electron mobilities of the devices with different Au-silica nanorod concentrations extracted from dark $J-V$ curves.

\begin{tabular}{lll}
\hline $\begin{array}{l}\text { Au NPs } \\
\begin{array}{l}\text { concentration } \\
(\text { wt\% })\end{array}\end{array}$ & $\begin{array}{l}\text { Zero-field hole mobility } \\
\left(\mathrm{cm}^{2} \mathrm{~V}^{-1} \mathrm{~s}^{-1}\right)\end{array}$ & $\begin{array}{l}\text { Zero-field electron mobility } \\
\left(\mathrm{cm}^{2} \mathrm{~V}^{-1} \mathrm{~s}^{-1}\right)\end{array}$ \\
\hline 0 & $1.2 \times 10^{-3}$ & $1.5 \times 10^{-3}$ \\
1 & $2.4 \times 10^{-3}$ & $2.5 \times 10^{-3}$ \\
2 & $1.6 \times 10^{-3}$ & $1.7 \times 10^{-3}$ \\
5 & $8.5 \times 10^{-4}$ & $9.0 \times 10^{-4}$ \\
\hline
\end{tabular}

local field and the incident field [30]. This performance measure of plasmonic materials is greatly determined by wavelength dependent complex dielectric function $(\varepsilon(\omega))$, where the real part $\left(\varepsilon_{1}(\omega)\right)$ describes the polarization induced by the electric field and imaginary part $\left(\varepsilon_{2}(\omega)\right)$ describes the energy losses that occur during the polarization in the materials $[31,32]$. Therefore, performance of plasmonic material becomes good when the material has large negative value of $\varepsilon_{1}(\omega)$ and small $\varepsilon_{2}(\omega)$ over the wavelength of application [31,33]. The quality factor for LSPR in metallic NPs $\left(Q_{L S P R}\right)$ largely depends on the shape of metallic NPs [30].

For a metallic nanosphere embedded within a silica matrix, the $Q_{L S P R}$ is defined as:

$Q_{L S P R(\text { nanosphere })} \equiv \frac{\left[\left(\varepsilon_{1}-3.9\right)^{2}+\varepsilon_{2}^{2}\right]^{1 / 2}}{\varepsilon_{2}}$

For a metallic rod-shaped nanospheroid embedded within a silica matrix, the $Q_{L S P R}$ is defined as:

$Q_{L S P R(\text { nanospheroid) }} \equiv \frac{1}{P_{A}} \frac{\left[\left(\varepsilon_{1}-3.9\right)^{2}+\varepsilon_{2}^{2}\right]^{1 / 2}}{\varepsilon_{2}}$

The derivation for both (1) and (2) can be found in Supplementary information. The parameter $P_{A}$ in (2) is the depolarization factor of the nanospheroid along the (major) $a$-axis and for the Au-silica nanorods used in this study, $P_{A}=0.12$. The complex refractive indexes at different wavelengths as tabulated by Johnson and Christy are used for calculation of the real part and imaginary part of the complex dielectric function [34]. The relation between the complex dielectric function and complex refractive index can be expressed as [31]:

$\varepsilon_{1}(\omega)=n(\omega)^{2}-k(\omega)^{2} ; \quad \varepsilon_{2}(\omega)=2 n(\omega) k(\omega)$

where $n(\omega)$ is refractive index, $k(\omega)$ is extinction coefficient.

Fig. 4a illustrates the complex dielectric function of $\mathrm{Au}$ which was calculated using the Eq. (3). It is observed that the $\varepsilon_{1}$ is negative in the visible and infrared range of $400-1000 \mathrm{~nm}$. In addition, there is small energy loss within the range of $600-1000 \mathrm{~nm}$. Therefore, it explains why Au NPs are usually used in the LSPR studies and application in the OSCs for light trapping. Fig. $4 \mathrm{~b}$ and Table S2 shows the $Q_{L S P R}$ for both Au-silica sphere and rod-shaped spheroid with the wavelength range from $400 \mathrm{~nm}$ to $1000 \mathrm{~nm}$. Since nanorod has good approximation to rod-shaped spheroid, it is clearly shown that Au-silica nanorod has higher $Q_{L S P R}$ than that for Au-silica nanosphere from $550 \mathrm{~nm}$ onward, which is in accordance with the major absorption spectrum of p-DTS(FBTTh $)_{2}$ donor. Therefore, Au-silica nanorod achieves larger localized field enhancement within the SM BHJ layer and is considered as better plasmonic material than Au-silica nanosphere.

On the other hand, light scattering effect by metallic NPs, especially large-sized NPs, plays an important role in the absorption enhancement of SM devices [25]. A more in-depth investigation was carried out by haze factor measurement which can examine the scattering effect [35]. Fig. 5 shows the haze factor spectra of transmittance $\left(H_{T}\right)$ and reflection $\left(H_{R}\right)$ calculated by the fraction 


\section{(a)}
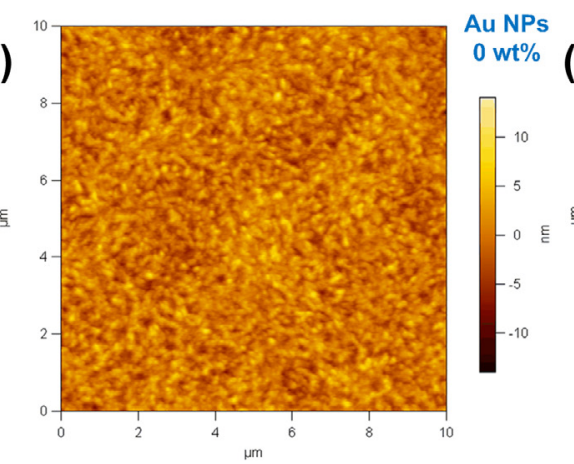

\section{(c)}
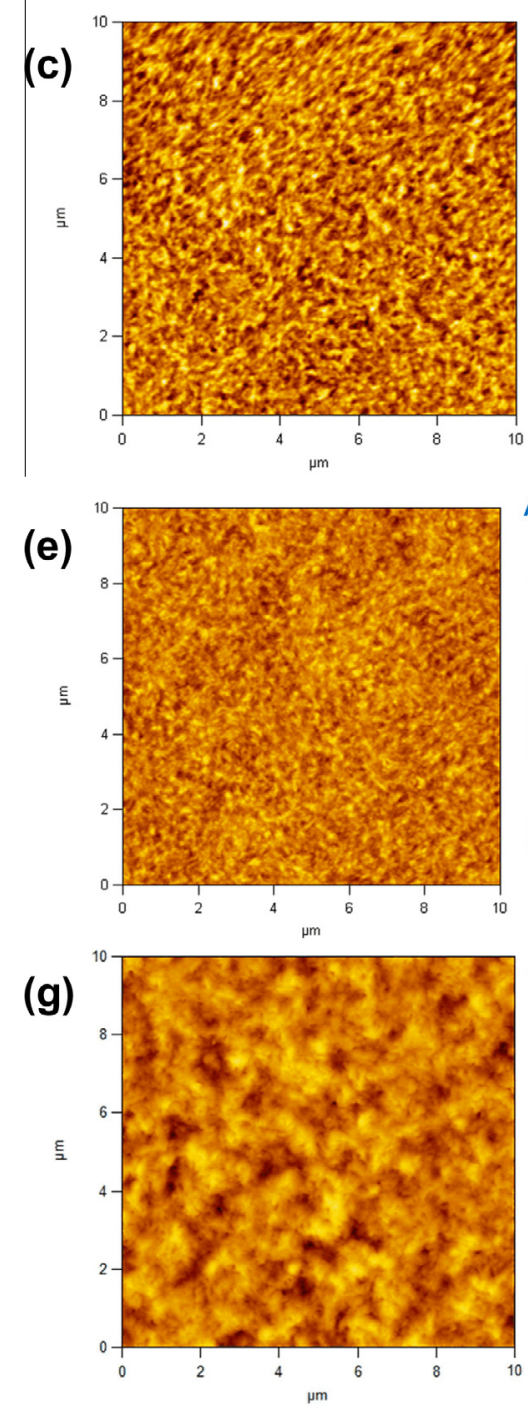

Au NPs

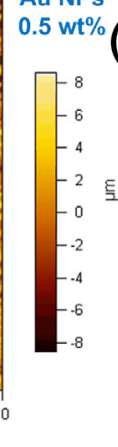

Au NPs

Au NPs

\section{(b)}

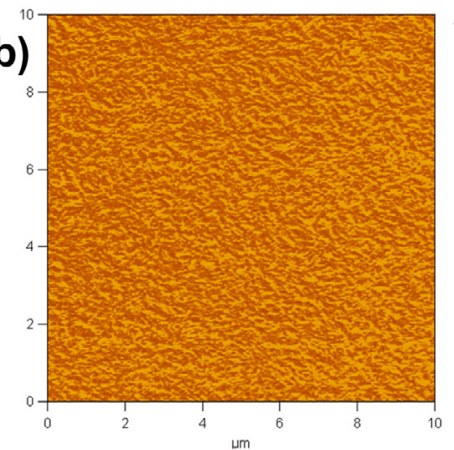

(d)

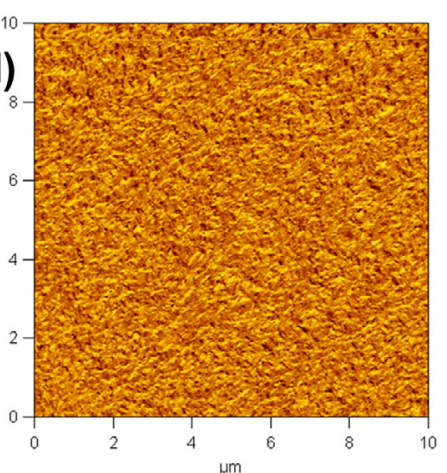

Au NPs

$0.5 w t \%$

$1 w t \%$ (f)

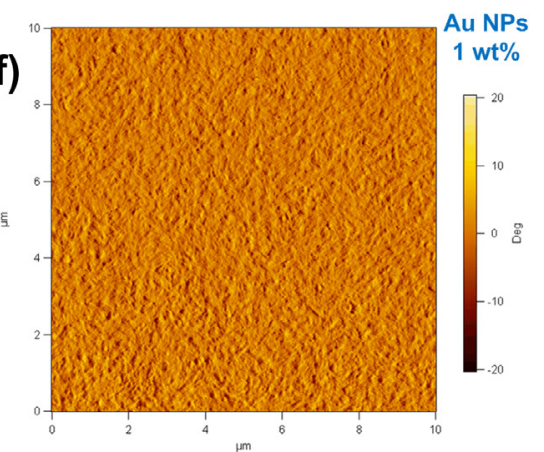

(h)

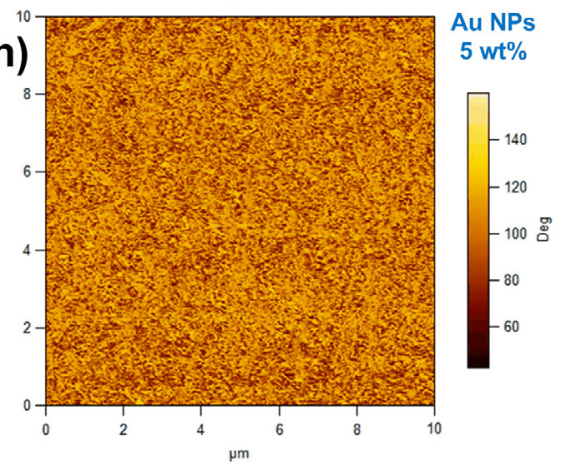

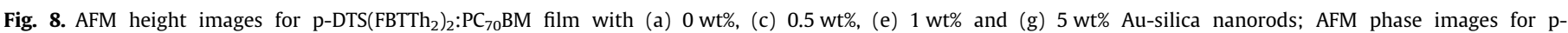
DTS(FBTTh $)_{2}: \mathrm{PC}_{70} \mathrm{BM}$ film with (b) $0 \mathrm{wt} \%$, (d) $0.5 \mathrm{wt} \%$, (f) $1 \mathrm{wt} \%$ and (h) $5 \mathrm{wt} \%$ Au-silica nanorods.

(in percent) of the diffuse transmittance/reflection relative to the total transmittance/reflection for p-DTS(FBTTh $)_{2}: \mathrm{PC}_{70} \mathrm{BM}$ film with and without Au-silica nanorods/nanospheres. $H_{T}$ and $H_{R}$ of plasmonic device with Au-silica nanospheres are very similar to that of reference device (without NPs) whereas significantly higher $H_{T}$ and $H_{R}$ are observed in plasmonic device with Au-silica nanorods in the range of wavelength $400-800 \mathrm{~nm}$. The results reveal that Au-silica nanorods have strong light scattering and thus contribute to more light absorption of SM BHJ compared to Au-silica nanospheres. These results are also consistent with the scattering cross section equation of metallic NPs which depends on the size and shape of NPs $[19,36,37]$. Generally, small NPs with spherical shape contribute negligible scattering to enhance the optical path length. On the contrary, for large metallic NPs $(\sim 100 \mathrm{~nm})$ with rod shape within the BHJ layer, the incident photons can be scattered more strongly by metallic NPs into a longer propagation path in the active layer. Taking the account together with above mentioned $Q_{L S P R}$, the incorporation of Au-silica nanorods into the SM 


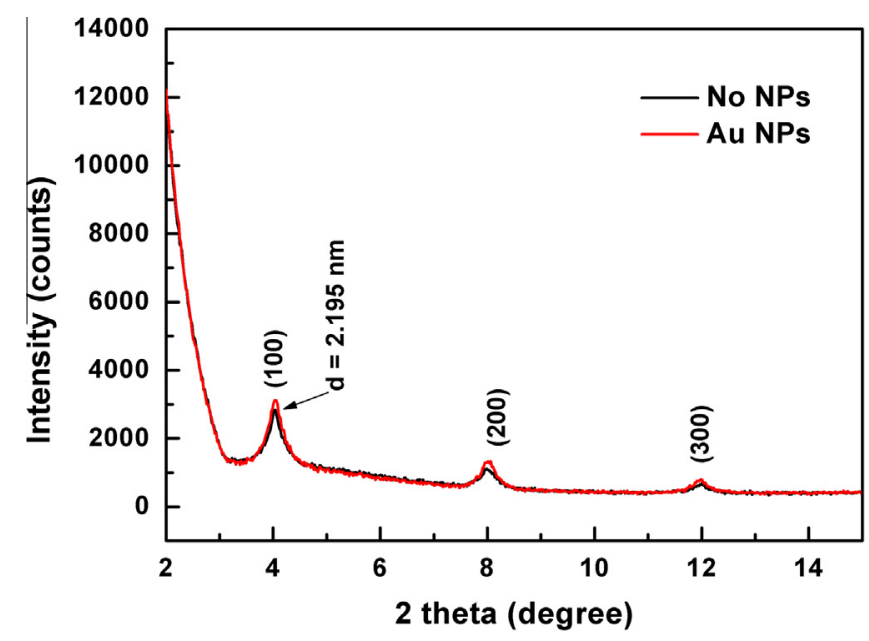

Fig. 9. Out-of-plane XRD spectra of both SM BHJ films without and with 1 wt\% of Au-silica nanorods.

BHJ device has superior performance in both LSPR effect and scattering effect compared with the Au-silica nanospheres.

\subsection{Effect of Au-silica nanorod concentration}

The possible role of the $\mathrm{BHJ}$ morphology change in the enhancement of PCE following incorporation of nanorods was also investigated by varying the concentration of Au-silica nanorods in the $\mathrm{BHJ}$. Fig. $6 \mathrm{a}$ and $\mathrm{b}$ represents the $J-V$ characteristics and the EQE spectra of the devices with the weight ratios of $0 \mathrm{wt} \%, 0.5 \mathrm{wt} \%$, $1 \mathrm{wt} \%, 2 \mathrm{wt} \%$ and $5 \mathrm{wt} \%$ of Au-silica nanorods. The average photovoltaic parameters calculated from ten devices for each weight percentage are listed in Table 1. After incorporation of Au-silica nanorods, the PCE increases with higher concentration of Au-silica nanorods and reaches a maximum at $1 \mathrm{wt} \%$. The highest PCE of $8.2 \pm 0.12 \%$ with $26.1 \%$ enhancement was achieved. The improvement in PCE is mainly due to increased $J_{S C}$ and was further confirmed by the EQE spectra. The EQE increases the most within the wavelength range from $450 \mathrm{~nm}$ to $700 \mathrm{~nm}$, which is wellmatched with the broad absorption range of dual plasmonic Au-silica nanorods. Despite significant changes in $J_{S C}, V_{O C}$ remains at $0.77 \mathrm{~V}$, implying that Au-silica nanorods do not change the nature of the electrode and the $\mathrm{BHJ}$ layer interface and the energy offsets between the respective HOMO and LUMO of p-DTS(FBTTh $)_{2}$ and $\mathrm{PC}_{70} \mathrm{BM}$. The similar $F F$ also suggests that Au-silica nanorods do not adversely affect the charge collection/recombination and carrier transport in the device. At the highest Au-silica nanorod concentration of $5 \mathrm{wt} \%$, however, the PCE of the devices declined because of decrease in $J_{S C}$ and $F F$.

The mobility of charge carriers is important for organic photovoltaic device performance because it needs to be high enough to give a high $F F, J_{S C}$ and PCE. When nanorods are blended into the active layer, there may be increased carrier scattering resulting in reduced mobility. It is therefore important to study the effect of nanorod concentration on the carrier transport in the active layer. By using electrodes which suppress either electrons or holes, we measured dark $J-V$ characteristics of hole-only and electrononly devices and numerically fitted the results using the spacecharge limited current (SCLC) model $[38,39]$. The Mott-Gurney law that includes a field dependent carrier mobility, is given by [40]:

$J=\frac{9}{8} \varepsilon_{0} \varepsilon_{r} \mu_{0} \frac{V^{2}}{L^{3}} \exp \left(\beta \sqrt{\frac{V}{L}}\right)$ where $\varepsilon_{0}$ is the permittivity of free space, $\varepsilon_{r}$ is the dielectric constant of the SM and fullerene blend; $\mu_{0}$ is the zero-field mobility; $V$ is the voltage drop across the BHJ layer which is the applied voltage $V_{\text {applied }}$ compensated by the built-in voltage $\left(V_{\mathrm{BI}}\right)$ and the voltage across the contacts $\left(V_{\mathrm{RS}}\right)$; i.e., $V=V_{\text {applied }}-V_{\mathrm{BI}}-V_{\mathrm{RS}} L$ is the thickness of the active layer and $\beta$ is the field activation factor.

The dark $J-V$ curves of hole-only and electron-only devices with Au-silica nanorod concentrations of $0 \mathrm{wt} \%, 1 \mathrm{wt} \%, 2 \mathrm{wt} \%$ and $5 \mathrm{wt} \%$ are shown in Fig. 7a and b respectively. The extracted zero-field hole and electron mobilities of reference device from these curves are $1.2 \times 10^{-3} \mathrm{~cm}^{2} \mathrm{~V}^{-1} \mathrm{~s}^{-1}$ and $1.5 \times 10^{-3} \mathrm{~cm}^{2} \mathrm{~V}^{-1} \mathrm{~s}^{-1}$ respectively, as shown in Table 2. Upon the incorporation of $1 \mathrm{wt} \%$ Au-silica nanorods, the zero-field hole and electron mobilities slightly increase to $2.4 \times 10^{-3} \mathrm{~cm}^{2} \mathrm{~V}^{-1} \mathrm{~s}^{-1}$ and $2.5 \times 10^{-3} \mathrm{~cm}^{2} \mathrm{~V}^{-1} \mathrm{~s}^{-1}$ respectively. The values of $\beta$ are almost same in those devices. The ratio between electron mobility and hole mobility in p-DTS(FBTTh $)_{2}: \mathrm{PC}_{70} \mathrm{BM}$ blend are observed to decrease from 1.2 to 1.06 , indicating both higher and more balanced carrier mobilities in p-DTS $\left(\text { FBTTh }_{2}\right)_{2}: \mathrm{PC}_{70} \mathrm{BM}$ solar cell after incorporating $1 \mathrm{wt} \%$ Au-silica nanorods. Hence, the incorporation of $1 \mathrm{wt} \% \mathrm{Au}-$ silica nanorods does not adversely affect the charge transport in the active layer. However, at 2 wt\% Au-silica nanorod concentration, the zero-field hole and electron mobilities declined to $1.6 \times 10^{-3} \mathrm{~cm}^{2} \mathrm{~V}^{-1} \mathrm{~s}^{-1}$ and $1.7 \times 10^{-3} \mathrm{~cm}^{2} \mathrm{~V}^{-1} \mathrm{~s}^{-1}$ respectively. When Au-silica nanorod concentration continued to rise to $5 \mathrm{wt} \%$, the zero-field hole and electron mobilities decreased to $8.5 \times 10^{-4} \mathrm{~cm}^{2} \mathrm{~V}^{-1} \mathrm{~s}^{-1}$ and $9.0 \times 10^{-4} \mathrm{~cm}^{2} \mathrm{~V}^{-1} \mathrm{~s}^{-1}$ respectively, which corresponds to the decreased performance of SM solar cells with $5 \mathrm{wt} \%$ Au-silica nanorods.

Carrier mobility of these SM devices might be affected by changed nanoscale morphology of the $\mathrm{BHJ}$ film after incorporation of Au-silica nanorods. AFM height and phase images of the films with various concentrations of Au-silica nanorods (0 wt\%, $0.5 \mathrm{wt} \%, 1 \mathrm{wt} \%$ and 5 wt\%) are shown in Fig. 8. At low Au-silica nanorod concentration, the root mean square (RMS) roughness of the BHJ film are $2.112 \mathrm{~nm}, 2.393 \mathrm{~nm}$ and $2.477 \mathrm{~nm}$ for films with $0 \mathrm{wt} \%, 0.5 \mathrm{wt} \%$ and $1 \mathrm{wt} \%$ Au-silica nanorods, respectively and no obvious difference in phase separation can be observed. AFM images suggest that we can add the Au-silica nanorods as high as $1 \mathrm{wt} \%$ without affecting the morphology of the $\mathrm{BHJ}$ film. However, the RMS roughness drastically increases to $12.487 \mathrm{~nm}$ when the concentration is increased to $5 \mathrm{wt} \%$. Such change in morphology induced by high nanorod concentration affects the charge transport process and thus degrades both hole mobility and electron mobility in SM devices, which agree well with the above results as shown in Fig. 7.

In addition to surface morphology examined by AFM, XRD study also confirms that Au-silica nanorods with concentration up to $1 \mathrm{wt} \%$ does not affect the bulk morphology of BHJ film [41]. As shown in Fig. 9, the out-of-plane XRD spectra of both BHJ films without and with $1 \mathrm{wt} \%$ of Au-silica nanorods show multiple peaks at $2 \theta \approx 4^{\circ}, 8^{\circ}, 12^{\circ}$ which correspond to the scattering from (100), (200) and (300) planes with $d$ spacing of $2.195 \mathrm{~nm}$. The high intensity peak of first order scattering and subsequent higher order scatterings suggests that both $\mathrm{BHJ}$ films with and without nanorods have lamellar structure with high crystallinity. Besides the peak intensity, the positions and full width at half maximum (FWHM) of the peaks of the two films are also similar to each other, implying that both films have similar crystalline correlation length (CCL) and the spacing of lamella stacking. Using the Scherrer equation, the CCL (estimated crystalline size) is $\sim 25 \mathrm{~nm}$ for the both film. As shown in Fig. S3, an additional XRD scan was performed for $2 \theta$ between $5^{\circ}$ and $40^{\circ}$ and this too revealed no change in the diffraction spectrum for $1 \mathrm{wt} \%$ Au-silica nanorod and the reference sample. Since there is almost no change in XRD results in optimized Au-silica nanorod concentration, it is confirmed that the addition of small amount of Au-silica does not affect the bulk 

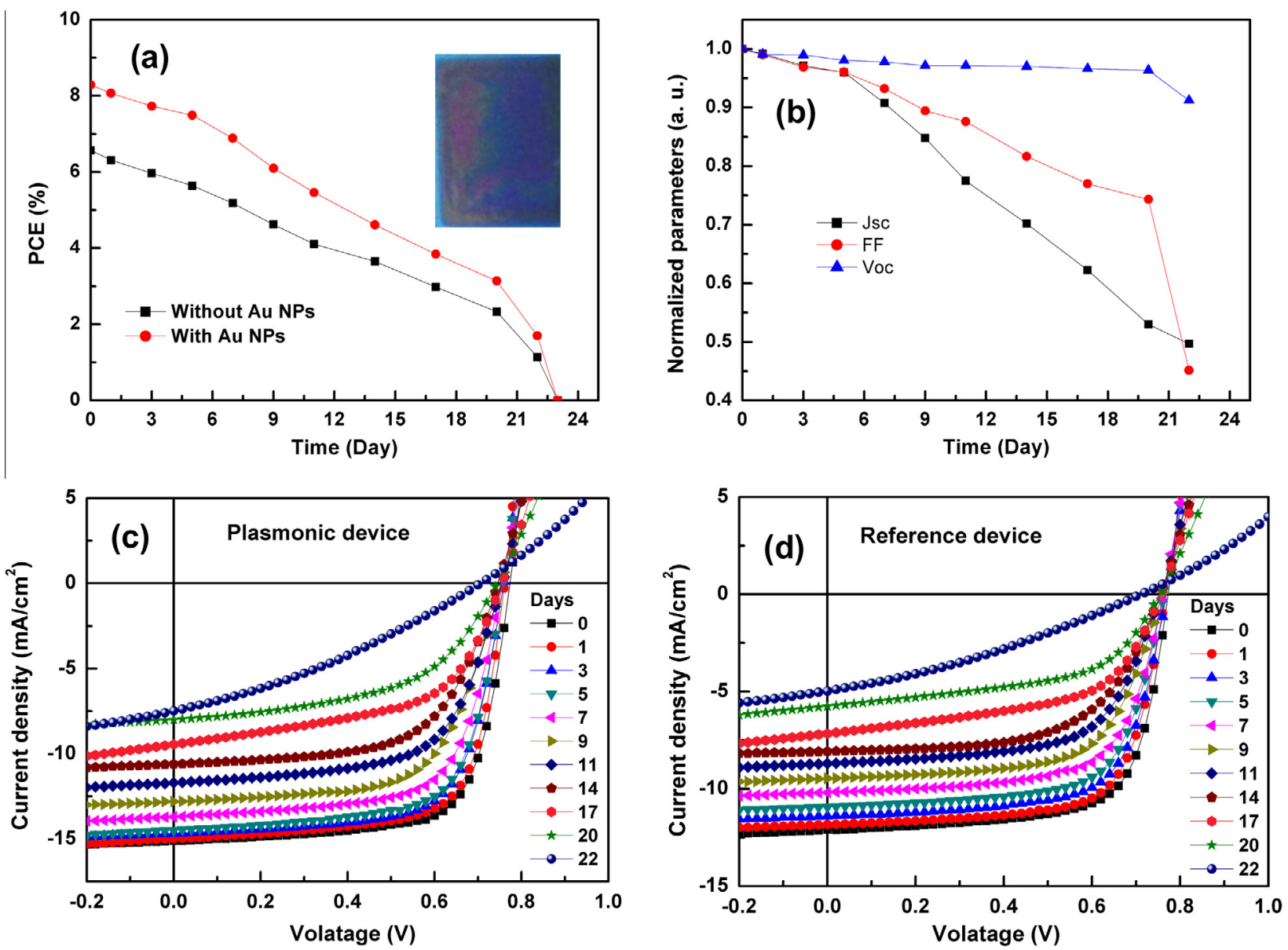

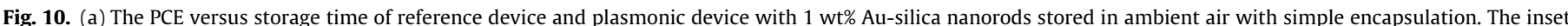

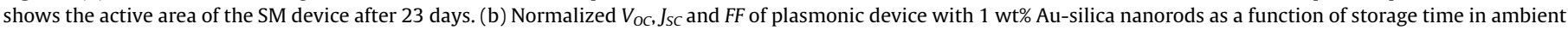

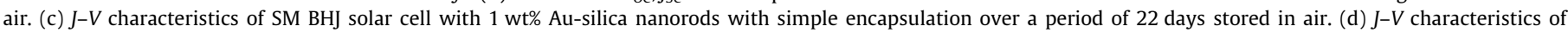
reference SM BHJ solar cell with simple encapsulation over a period of 22 days stored in air.

morphology. In order to explain the increase in electron and hole mobility of SM device with $1 \mathrm{wt} \%$ nanorods, we propose that the Au-silica nanorods provide an alternative hopping conduction pathway for the dissociated charge carriers [42]. The thickness of the silica shell is in a range where direct tunneling can occur. The energy band diagram of the p-DTS $\left(\mathrm{FBTTh}_{2}\right)_{2}, \mathrm{PC}_{70} \mathrm{BM}, \mathrm{SiO}_{2}$ and $A u$ are shown in Fig. S4. Indirect empirical evidence for tunneling of charge carriers on Au NPs with and without silica shell was obtained via carrier recombination measurements performed using light intensity dependence of the short-circuit current density [24]. The value of $S=0.913, S=0.944$ and $S=0.971$ for bare Au nanorods, Au nanorods with $5 \mathrm{~nm}$ silica shell and Au nanorods with $10 \mathrm{~nm}$ silica shell respectively were determined by fitting the data with a power law dependence on the illumination light intensity equation: $J_{S C} \propto P^{S}$. Usually, when the value of $S$ is equal to 1 , it means that there is no bimolecular recombination and space-limited charges for ideal material without defects. Since the values of $S$ for bare Au nanorods, Au nanorods with $5 \mathrm{~nm}$ silica shell and Au nanorods with $10 \mathrm{~nm}$ silica shell are all less than 1, there is bimolecular recombination in all devices. However, there is less bimolecular recombination for the device with Au nanorods with $5 \mathrm{~nm}$ silica shell than the bare Au nanorod with no silica shell. In turn the device with Au nanorods with $10 \mathrm{~nm}$ silica shell has less bimolecular recombination than the device with Au nanorods with $5 \mathrm{~nm}$ silica shell. This showed that there is still some carrier recombination and thus the tunneling of charge carrier existed in such thin silica shell. Combined with above analysis, the PCE enhancement in plasmonic SM device results from light scattering and LSPR effect rather than the morphology change of the SM BHJ layer induced by Au-silica nanorods.

\subsection{Study of the device stability}

Finally, we studied the stability of reference and plasmonic devices because the stability or degradation characteristic is as important as the efficiency and we have to ensure that Au-silica nanorods used for efficiency enhancement should not have negative impact on the stability of the device. Furthermore, the influence of plasmonic metallic NPs on the device stability has never been reported before. Due to the nature of the organic active layer and interfacial layers, OSCs typically degrade rapidly when exposed to $\mathrm{O}_{2}$ and moisture at ambient condition. Therefore, we used a simple encapsulation scheme using epoxy and cover glass to test the effect of Au-silica nanorods on the stability of solution-processed SM BHJ solar cells. Fig. 10a shows the PCE as a function of storage time in air of the encapsulated reference device and plasmonic device with $1 \mathrm{wt} \%$ nanorods. After 20 days in ambient air, the PCE of reference and plasmonic devices show similar trends and finally decrease to $35.5 \%$ and $37.9 \%$ of the initial PCE values, respectively, indicating that incorporation of Au-silica nanorods into device has little effect on the stability of SM solar cells. The changes in normalized $V_{O C}, J_{S C}$ and $F F$ of plasmonic device are shown in Fig. 10b. The $V_{O C}$ has a slight decrease from $0.774 \mathrm{~V}$ to $0.746 \mathrm{~V}$ whereas both the normalized $J_{S C}$ and $F F$ have a large 
decrease to $53.0 \%$ and $74.3 \%$ of initial values, respectively, after 20 days. However, the PCE in both the devices with and without $1 \mathrm{wt} \%$ nanorods dropped quickly in the 22 days and finally reduced to zero in the 23 days because the $\mathrm{O}_{2}$ and moisture in air have percolated into the devices and thus caused the rapid degradation of the device performance as shown in the inset of Fig. 10a. The detailed $J-V$ characteristics of SM BHJ solar cells with and without $1 \mathrm{wt} \%$ Au-silica nanorods with simple encapsulation over a period of 22 days stored in air are shown in Fig. 10c and d.

\section{Conclusions}

We improved the PCE of solution-processed SM solar cells by $\sim 5 \%$ and $\sim 26 \%$ upon incorporation of Au-silica nanospheres and nanorods respectively into p-DTS( $\left.\mathrm{FBTTh}_{2}\right)_{2}: \mathrm{PC}_{70} \mathrm{BM}$ active layer. The PCE enhancement in the device incorporated with nanorods is significantly higher than that in the device incorporated with nanospheres. The higher PCE enhancement is originated from the stronger scattering effect and higher quality factor for LSPR of rod-shaped NPs. XRD and AFM topography show that there is almost no changes in both surface and bulk morphology of $\mathrm{BHJ}$ when incorporating the optimum concentration (1 wt\%) of Au-silica nanorods. In addition, high electron and hole mobilities with balanced charge transports are maintained at the optimum concentration of nanorods. Finally, the lifetime studies of the SM solar cells show that the addition of Au-silica nanorods has little effect on the device stability.

\section{Acknowledgements}

A.K.K.K. thanks the support of A*STAR's Science and Engineering Research Council through TSRP grant (Grant \#102 170 0137). Q.X., H.V.D., and X.W.S. gratefully thank the support from Singapore National Research Foundation through a Competitive Research Program (NRF-CRP-6-2010-2). X.W.S. would like to acknowledge the support from Singapore National Research Foundation (NRF-CPR11-2012-01), New Initiative Fund and JOINT SINGAPOREAN-GERMAN RESEARCH PROJECTS from Nanyang Technological University.

\section{Appendix A. Supplementary data}

Supplementary data associated with this article can be found, in the online version, at http://dx.doi.org/10.1016/j.orgel.2015.03. 026.

\section{References}

[1] Y. Yang, J. Zhang, Y. Zhou, G. Zhao, C. He, Y. Li, M. Andersson, O. Inganäs, F. Zhang, J. Phys. Chem. C 114 (2010) 3701-3706.

[2] B. Yin, L. Yang, Y. Liu, Y. Chen, Q. Qi, F. Zhang, S. Yin, Appl. Phys. Lett. 97 (2010) 023303.
[3] Y. Sun, G.C. Welch, W.L. Leong, C.J. Takacs, G.C. Bazan, A.J. Heeger, Nat. Mater. 11 (2012) 44-48.

[4] A.K.K. Kyaw, D.H. Wang, D. Wynands, J. Zhang, T.-Q. Nguyen, G.C. Bazan, A.J. Heeger, Nano Lett. 13 (2013) 3796-3801.

[5] T.S. van der Poll, J.A. Love, T.-Q. Nguyen, G.C. Bazan, Adv. Mater. 24 (2012) 3646-3649.

[6] V. Gupta, A.K.K. Kyaw, D.H. Wang, S. Chand, G.C. Bazan, A.J. Heeger, Sci. Rep. 3 (2013) 1965.

[7] S.R. Forrest, MRS Bull. 30 (2005) 28-32.

[8] H. Hoppe, N.S. Sariciftci, J. Mater. Res. 19 (2004) 1924-1945.

[9] C.J. Brabec, Sol. Energy Mater. Sol. Cells 83 (2004) 273-292.

[10] Z. Tang, W. Tress, O. Inganäs, Mater. Today 17 (2014) 389-396.

[11] E. Stratakis, E. Kymakis, Mater. Today 16 (2013) 133-146.

[12] S.V. Boriskina, H. Ghasemi, G. Chen, Mater. Today 16 (2013) 375-386.

[13] P. Spinelli, A. Polman, Opt. Express 20 (2012) A641-A654.

[14] M. Xue, L. Li, B.J. Tremolet de Villers, H. Shen, J. Zhu, Z. Yu, A.Z. Stieg, Q. Pei, B.J. Schwartz, K.L. Wang, Appl. Phys. Lett. 98 (2011) 253302.

[15] J.-L. Wu, F.-C. Chen, Y.-S. Hsiao, F.-C. Chien, P. Chen, C.-H. Kuo, M.H. Huang, C.S. Hsu, ACS Nano 5 (2011) 959-967.

[16] L. Lu, Z. Luo, T. Xu, L. Yu, Nano Lett. 13 (2012) 59-64.

[17] F.X. Xie, W.C.H. Choy, C.C.D. Wang, W.E.I. Sha, D.D.S. Fung, Appl. Phys. Lett. 99 (2011) 153304.

[18] D.D.S. Fung, L. Qiao, W.C.H. Choy, C. Wang, W.E.I. Sha, F. Xie, S. He, J. Mater. Chem. 21 (2011) 16349-16356.

[19] K.S. Lee, M.A. El-Sayed, J. Phys. Chem. B 109 (2005) 20331-20338.

[20] X. Xu, A.K.K. Kyaw, B. Peng, D. Zhao, T.K.S. Wong, Q. Xiong, X.W. Sun, A.J. Heeger, Org. Electron. 14 (2013) 2360-2368.

[21] R.C. Wadams, C.-W. Yen, D.P. Butcher Jr., H. Koerner, M.F. Durstock, L. Fabris, C.E. Tabor, Org. Electron. 15 (2014) 1448-1457.

[22] T. Ming, L. Zhao, Z. Yang, H. Chen, L. Sun, J. Wang, C. Yan, Nano Lett. 9 (2009) 3896-3903.

[23] B. Peng, Q. Zhang, X. Liu, Y. Ji, H.V. Demir, C.H.A. Huan, T.C. Sum, Q. Xiong, ACS Nano 6 (2012) 6250-6259.

[24] X. Xu, Q. Du, B. Peng, Q. Xiong, L. Hong, H.V. Demir, T.K.S. Wong, A.K.K. Kyaw, X.W. Sun, Appl. Phys. Lett. 105 (2014) 113306.

[25] D.H. Wang, D.Y. Kim, K.W. Choi, J.H. Seo, S.H. Im, J.H. Park, O.O. Park, A. Heeger, Angew. Chem. Int. Ed. 50 (2011) 5519-5523.

[26] H. Choi, J.P. Lee, S.J. Ko, J.W. Jung, H. Park, S. Yoo, O. Park, J.R. Jeong, S. Park, J.Y. Kim, Nano Lett. 13 (2013) 2204-2208.

[27] C.C.D. Wang, W.C.H. Choy, C. Duan, D.D.S. Fung, W.E.I. Sha, F.X. Xie, F. Huang, Y. Cao, J. Mater. Chem. 22 (2012) 1206-1211.

[28] K.R. Catchpole, A. Polman, Opt. Express 16 (2008) (1800) 21793-21800.

[29] X. Xu, A.K.K. Kyaw, B. Peng, Q. Du, L. Hong, H.V. Demir, T.K.S. Wong, Q. Xiong, X.W. Sun, Chem. Commun. 50 (2014) 4451-4454.

[30] P.R. West, S. Ishii, G.V. Naik, N.K. Emani, V.M. Shalaev, A. Boltasseva, Laser Photon. Rev. 4 (2010) 795-808.

[31] C.D. Louis, O. Pluchery, Gold Nanoparticles for Physics, Chemistry and Biology, Imperial College Press, London; Distributed by World Scientific Pub. Co., Singapore, 2012.

[32] A. Rastar, M. Esmail Yazdanshenas, A. Rashidi, S. Mansour Bidoki, J. Eng. Fibers Fabr. 8 (2013) 85-96.

[33] I.D. Mayergoyz, World Scientific Series in Nanoscience and Nanotechnology, Singapore, 2013.

[34] P.B. Johnson, R.W. Christy, Phys. Rev. B 6 (1972) 4370-4379.

[35] Y.-S. Hsiao, C.-P. Chen, C.-H. Chao, W.-T. Whang, Org. Electron. 10 (2009) 551561.

[36] S. Link, M.B. Mohamed, M.A. El-Sayed, J. Phys. Chem. B 103 (1999) 3073-3077.

[37] S. Link, M.A. El-Sayed, J. Phys. Chem. B 103 (1999) 8410-8426.

[38] V.D. Mihailetchi, L.J.A. Koster, J.C. Hummelen, P.W.M. Blom, Phys. Rev. Lett. 93 (2004) 216601.

[39] Y. Liang, Z. Xu, J. Xia, S.T. Tsai, Y. Wu, G. Li, C. Ray, L. Yu, Adv. Mater. 22 (2010) E135-E138.

[40] P.N. Murgatroyd, J. Phys. D Appl. Phys. 3 (1970) 151.

[41] A.K.K. Kyaw, D.H. Wang, V. Gupta, W.L. Leong, L. Ke, G.C. Bazan, A.J. Heeger, ACS Nano 7 (2013) 4569-4577.

[42] M. Xiue, L. Li, B.J. Tremolet de Villers, H. Shen, J. Zhu, Z. Yu, A.Z. Stieg, Q. Pei, B.J. Schwartz, K.L. Wang, Appl. Phys. Lett. 98 (2011) 253302. 